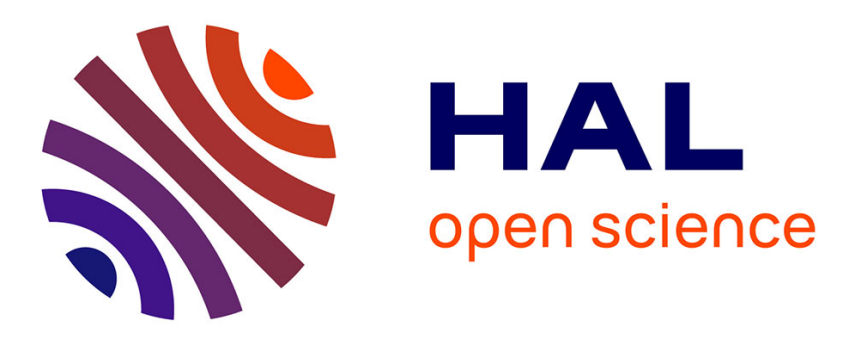

\title{
Deploying Fast-charging Stations for Electric Vehicles Based on Mobility Flows and Local Photovoltaic Production
}

\author{
Abood Mourad, Martin Hennebel, Ahmed Amrani, Amira Ben Hamida
}

\section{To cite this version:}

Abood Mourad, Martin Hennebel, Ahmed Amrani, Amira Ben Hamida. Deploying Fast-charging Stations for Electric Vehicles Based on Mobility Flows and Local Photovoltaic Production. 2020 17th International Conference on the European Energy Market (EEM), Sep 2020, Stockholm, Sweden. 10.1109/EEM49802.2020.9221948 . hal-02958631

\section{HAL Id: hal-02958631 \\ https://hal.science/hal-02958631}

Submitted on 6 Oct 2020

HAL is a multi-disciplinary open access archive for the deposit and dissemination of scientific research documents, whether they are published or not. The documents may come from teaching and research institutions in France or abroad, or from public or private research centers.
L'archive ouverte pluridisciplinaire HAL, est destinée au dépôt et à la diffusion de documents scientifiques de niveau recherche, publiés ou non, émanant des établissements d'enseignement et de recherche français ou étrangers, des laboratoires publics ou privés. 


\title{
Deploying Fast-charging Stations for Electric Vehicles Based on Mobility Flows and Local Photovoltaic Production
}

\author{
Abood Mourad*, Martin Hennebel*, Ahmed Amrani ${ }^{\dagger}$ and Amira Ben Hamida ${ }^{\dagger}$ \\ * Laboratoire Génie électrique et électronique de Paris, CentraleSupélec \\ Université Paris-Saclay, Gif-sur-Yvette, France \\ Email: abood.mourad@centralesupelec.fr, martin.hennebel@centralesupelec.fr \\ ${ }^{\dagger}$ Institut de Recherche Technologique SystemX, Palaiseau, France \\ Email: Ahmed.AMRANI@irt-systemx.fr , Amira.BENHAMIDA@irt-systemx.fr
}

\begin{abstract}
The need for deploying fast-charging stations for electric vehicles (EVs) is becoming essential in recent years. This need is justified by the increasing recharging demand, and supported by new recharging technologies making EV chargers more efficient. For this purpose, we introduce an optimization model for finding the optimal deployment of EV fast-charging stations through a network of highways, while taking into account their recharging demands and the restrictions imposed by the electric grid. We also consider the availability of local photovoltaic (PV) power station and integrate its energy to the proposed recharging network. In addition, we present a case study on Paris-Saclay area where the actual mobility flows and electric network specifications are considered. We thus provide the optimal locations for EV charging stations at the studied area, and we indicate the number of chargers to be deployed at each station. Finally, we study the potential benefits of integrating local PV energy and analyze its different prices and production costs.
\end{abstract}

Index Terms-electric vehicles, recharging, optimization, mobility, photovoltaic

\section{INTRODUCTION}

Electric Vehicles (EVs) represent a promising opportunity that can lead to fundamental shifts in people transportation. With their batteries and charging times being considerably enhanced in recent years, EVs can provide a sustainable transportation solution [1]. The deployment of fast-charging stations is thus essential especially on highways where EVs need to be recharged at very short periods of time. For this purpose, chargers with high powers need to be installed in order to fulfil the increasing charging demand for EVs. In addition, the availability of locally-produced green energy (e.g. wind or solar sources) can also help in providing the energy needed to supply these chargers. However, green energy sources still suffer from uncertainty in their production and their relatively high production costs [2].

In this paper, we introduce an optimization model to find the optimal locations for deploying EV fast-charging stations through a network of highways. The model takes into account

978-1-7281-6919-4/20/\$31.00 @2020 IEEE
EV charging demands as well as the capacity provided by the electric grid. In addition, we consider the availability of local photovoltaic (PV) power station and we study the use of its produced energy for supplying EV chargers. We thus present a mathematical formulation for the problem where the aim is to maximize charging demand coverage while respecting budget limits, parking slot limitations, and the available capacities of the electric grid and PV production. Then, we present a case study on Paris-Saclay area where the optimal locations for EV charging stations at the studied area are provided as well as the number of fast-chargers at each location. Finally, we study the potential benefits of integrating locally-produced PV energy and analyze its different prices and production costs using different investment indices.

\section{RELATED WORK}

Research on deploying EV fast-charging stations on highspeed roads is swiftly increasing in recent years. These fast chargers, also referred to as level-3 chargers, are characterized by their high charging capacity (400-500V/50A) and relatively short charging times (e.g. a Tesla Roadster can be fully recharged in less than 20 minutes) [3]. For example, in [4], the problem of selecting locations for fast-charging stations through a highway network for long-distance trips in the US was considered. Through a mixed integer programming model, authors efficiently selected the number and locations of EV charging stations and highlighted the importance of this selection in planning such a charging service. Another fast-charging location model was introduced in [5], where the selection of charging locations was based on passenger travel behaviors as well as the specifications of the electric grid. Through a case study on Dalian-China, the authors concluded that travel distances and located charging capacities are key factors in successfully deploying fast-charging services.

Many recent studies are focusing on using green energy sources for supplying EV charging networks. In [6], a review of photovoltaic energy systems along with their sizing strategies, optimization techniques, and cost evaluation methodologies was provided. In addition, the main indices for analyzing 
energy costs and pricing schemes has been presented. A relevant application of these indices can be found in [7] where the investment of a photovoltaic energy system was evaluated through a case study in three different cities in Turkey. In order to model production uncertainty in photovoltaic energy systems, a scenario-based approach to evaluate the potential use of solar energy for charging EVs was introduced in [8]. Results of testing the proposed approach on two Scandinavian cities demonstrated that PV energy yield can cover EV demands in most of the considered scenarios. The necessity of stochastic approaches to deal with uncertainty in PV production was also confirmed in [9]. This was done by evaluating a set of case studies using a linear programming framework that takes into account PV uncertainty and stochastic EV driving schedules.

\section{PROBLEM DESCRIPTION}

In this problem, we consider two types of fast chargers, one for charging small electric vehicles, and the other for charging heavy electric trucks. In addition, We consider a set of potential charging locations $\mathcal{S}=\mathcal{I} \cup \mathcal{V}$, where $\mathcal{I}$ includes locations that are powered by the distribution network, and $\mathcal{V}$ includes those that are powered by a local PV station. Every charging location $s \in \mathcal{S}$ is defined by a cost $\left(c_{s}\right)$ indicating required investment to use this location as a charging station, and has a maximum electric capacity and a max/min number of fast chargers that can be installed for small vehicles as well as heavy trucks $\left(\max _{s}^{a}, \min _{s}^{a}, \max _{s}^{b}\right.$ and $\min _{s}^{b}$ respectively). The investment cost $\left(c_{s}\right)$ includes land cost and cabling expenses. For charging locations that are powered by the distribution network, the maximum electric capacity is fixed and depends on the distribution network itself. We refer to this fixed capacity as $q_{s}(\forall s \in \mathcal{I})$. On the other hand, this capacity can not be fixed for charging locations that are powered by PV energy as it highly depends on PV energy production which is stochastic (i.e. it depends on cloudiness index, season, and solar irradiation). We thus refer to this varying electric capacity as $\lambda_{s}(\forall s \in \mathcal{V})$. In order to build mobility paths, we consider a set of coupling nodes $N$ representing different highway intersections. As such, we consider a set of paths $\mathcal{P}$ that link sequences of coupling nodes. Every path $p \in \mathcal{P}$ is defined by its charging demands $d_{p}^{a}, d_{p}^{b}$, representing the number of electric vehicles and trucks, respectively, to be recharged per day. The set of charging locations that are associated with each path $p$ is denoted as $S_{p}$. The numbers of electric vehicles and trucks that can be recharged using a fast charger per day are defined as $\beta^{a}$ and $\beta^{b}$ respectively. Similarly, the amounts of electric power needed to recharge an electric vehicle or an electric truck using a fast charger are defined as $q^{a}$ and $q^{b}$ respectively. Moreover, installing a fast charger for electric vehicles or trucks implies extra costs (i.e. charger costs). These costs are defined as $c^{a}$ and $c^{b}$ respectively. For the sake of simplicity, we assume all electric vehicles to be homogeneous, and thus, their recharging time and energy are the same. We consider the same assumption for electric trucks.

Hence, the choice of deploying a charging station at location $s \in \mathcal{S}$ and the number of fast chargers to be installed is based on the recharging demand through the path where $s$ is located. However, the number of chargers to be installed is limited by the available electric capacity and by the availability of places and parking slots at location $s$. As such, we build a model to optimize this deployment by maximizing the satisfied demand while respecting the different constraints.

\section{Mathematical Modeling}

In order to model the problem, we introduce a binary variable $x_{s}$ which is equal to 1 if a charging station is deployed at location $s \in \mathcal{S}$, and 0 otherwise. In addition, we introduce a continuous variable $y_{p} \in[0,1]$ representing the demand coverage rate on path $p$, and two integer variables, $z_{s}^{a}$ and $z_{s}^{b}$, representing the number of fast chargers to be installed at charging location $s \in \mathcal{S}$ for electric vehicles and trucks respectively. The optimization model is thus formulated as follows:

$\operatorname{Max} Z=\sum_{p \in \mathcal{P}}\left(d_{p}^{a}+d_{p}^{b}\right) y_{p}$

s.t.

$$
\begin{aligned}
& \sum_{s \in \mathcal{S}} c_{s} x_{s}+c^{a} z_{s}^{a}+c^{b} z_{s}^{b} \leq \mathcal{C} \\
& \sum_{p \in \mathcal{P}}\left(d_{p}^{a}+d_{p}^{b}\right) y_{p} \geq \mathcal{Q} \\
& \sum_{s \in \mathcal{S}_{p}} \beta^{a} z_{s}^{a} \geq d_{p}^{a} \quad \forall p \in \mathcal{P} \\
& \sum_{s \in \mathcal{S}_{p}} \beta^{b} z_{s}^{b} \geq d_{p}^{b} \quad \forall p \in \mathcal{P} \\
& \min _{s}^{a} \leq z_{s}^{a} \leq \max _{s}^{a} \quad \forall s \in \mathcal{S} \\
& \min _{s}^{b} \leq z_{s}^{b} \leq \max _{s}^{b} \quad \forall s \in \mathcal{S} \\
& q^{a} z_{s}^{a}+q^{b} z_{s}^{b} \leq q_{s} \quad \forall s \in \mathcal{I} \\
& q^{a} z_{s}^{a}+q^{b} z_{s}^{b} \leq \lambda_{s} \quad \forall s \in \mathcal{V} \\
& \text { if } y_{p}=0 \Longrightarrow z_{s}^{a}+z_{s}^{b}=0 \quad \forall s \in \mathcal{S}_{p}, \forall p \in \mathcal{P} \\
& \text { if } z_{s}^{a}+z_{s}^{b}>0 \Longrightarrow x_{s}=1 \quad \forall s \in \mathcal{S} \\
& x_{s} \in\{0,1\}, y_{p} \in[0,1] \quad \forall s \in \mathcal{S}, \forall p \in \mathcal{P}
\end{aligned}
$$




$$
\begin{aligned}
& z_{s}^{a}, z_{s}^{b}, q_{s}, \lambda_{s}, \in \mathcal{N} \quad \forall s \in \mathcal{S} \\
& d_{p}^{a}, d_{p}^{b}, \beta^{a}, \beta^{b}, q^{a}, q^{b} \in \mathcal{N} \quad \forall p \in \mathcal{P} \\
& \min _{s}^{a}, \max _{s}^{a}, \min _{s}^{b}, \max _{s}^{b} \in \mathcal{N} \quad \forall s \in \mathcal{S} \\
& c_{s}, c^{a}, c^{b} \in \mathcal{R} \quad \forall s \in \mathcal{S}
\end{aligned}
$$

The objective function (1) aims at maximizing the total covered demand for charging both vehicles and trucks. Regarding model constraints, constraint (2) ensures that the sum of location costs and chargers installation costs does not exceed total budget limit. Constraint (3) states that a minimum coverage of the overall charging demand must be ensured. Constraints 4 and 5 ) ensure that charging demands, for vehicles and trucks respectively, are covered at each mobility path. Constraints (6) and (7) state that the number of fast chargers to be installed must respect the specified limits at each charging location. Constraints (8) ensure that the electric power required to operate the installed chargers at a specific charging location does not exceed the available electric capacity provided by the distribution network at that location. Similarly, constraints 9 ensure that the electric power needed at charging locations, where a PV station is located nearby, must not exceed the actual PV energy production provided by that station. Constraints (10) and (11) are used to link the different variables of the model. Finally, constraints 12 to (16) define domains for model variables and parameters.

\section{RESUlts AND Discussion}

In this section, we introduce our case study for ParisSaclay area and describe how its different parameters and PV production scenarios are generated (section $\mathrm{V}-\mathrm{A}$ ). We then test the proposed model using Cplex solver and present the obtained results (section V-B). Finally, we study the different energy prices and production costs and analyze their impact on the profitability of the project (section $\mathrm{V}-\mathrm{C}$ ).

\section{A. Paris-Saclay case study}

Paris-Saclay is an agglomeration community located at the south of Paris. As it is geographically close to the capital and to main economical activities, Paris-Saclay is traversed by many major mobility axes (e.g. A6, A10, N20 in Fig. 1). These axes are used by personal vehicles and heavy trucks for short-distance trips (e.g. commuting) as well as international transit flows. In addition, a large photo-voltaic (PV) farm is to be installed at Marcoussis district (next to the intersection of "A10" and "N104" highways, Fig. 1) with 76500 solar panels and up to $24 \mathrm{GWh}$ annual power production. This farm has the potential of increasing charging power at nearby EV charging stations while reducing their reliance on the existing distribution network.

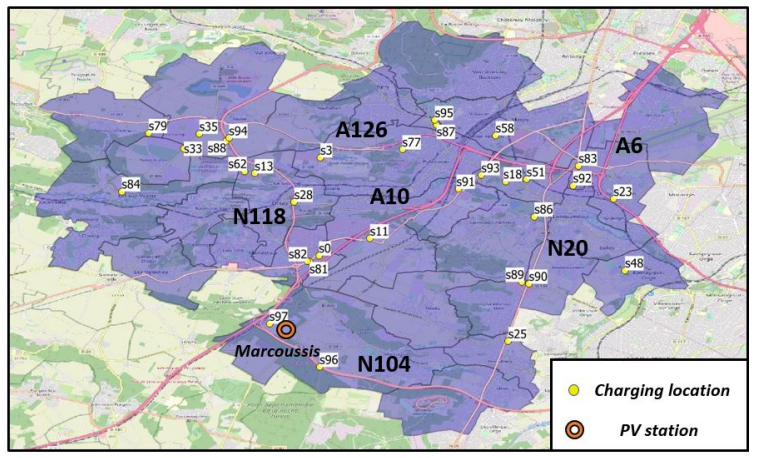

Fig. 1: Paris-Saclay - Major axes and recharging points

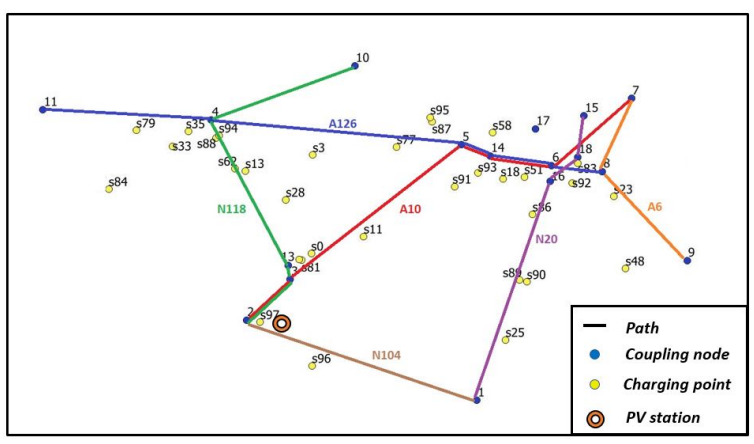

Fig. 2: Paris-Saclay - Mobility paths

In this study, we consider the intersections of major axes to be the set of coupling nodes. Mobility paths are then constructed using sequences of these nodes. For example, path "N20" is defined by the sequence of nodes $(1,16,18,15)$ (Fig. 2). As such, we define 9 different paths based on the actual mobility flows at Paris-Saclay area (Fig. 2). Each path is associated with vehicle and truck flows and their charging demands. The actual charging demand (i.e. the number of EVs to be recharged) is then calculated by considering $5 \%$ of the overall daily flow on each one of the defined paths 1 (i.e. an average of 2980 vehicle/h on each path). This percentage is fixed based on EV sales share of the French automobile market in 2020 [10]. In addition, we consider the actual charging stations (currently operational or projected) and the actual service stations as the set of potential charging locations at Paris-Saclay area. Costs, electric grid specifications, and the maximum number of fast chargers that can be installed at each location are fixed based on their real data ${ }^{2}$ Charging locations are then associated to paths so that the model can decide which locations to use and how many chargers to install at each location. Regarding chargers power, we assume that a fast charger requires $50 \mathrm{~kW}(\approx 20$ minutes $)$ for recharging an electric vehicle, and $250 \mathrm{~kW}$ ( $\approx 30$ minutes) for recharging an electric truck. The costs of installing a fast charger for vehicles

\footnotetext{
${ }^{1}$ Mobility flows at Paris-Saclay area are provided by DiRIF (Road Direction in Ile-de-France region) indicating number of vehicles per hour on each path.

${ }^{2}$ Data of charging stations and electric network specifications are provided by Paris-Saclay Agglomeration Community (CPS)
} 
and trucks are estimated at $12 \mathrm{k} €$ and $15 \mathrm{k} €$, respectively. Test parameters are summarized in Table $\mathbb{I}$.

TABLE I: Test parameters

\begin{tabular}{|c|c|c|c|}
\hline Parameter & Value & Parameter & Value \\
\hline$c^{a}$ & $12 \mathrm{k} €$ & $c^{b}$ & $15 \mathrm{k} €$ \\
$\beta^{a}$ & 36 vehicles & $\beta^{b}$ & 24 trucks \\
$q^{a}$ & $50 \mathrm{~kW}$ & $q^{b}$ & $250 \mathrm{~kW}$ \\
\hline
\end{tabular}

Regarding PV production, the generated power of a PV farm at instant $t$ can be calculated as follows:

$$
P(t)=(1-\mu(t)) \times I(t) \times \delta \times \eta
$$

Where $\mu(t)$ is the cloudiness index at instant $t(\%), I(t)$ is the irradiation at instant $t\left(W / m^{2}\right), \delta$ is the surface of solar panels $\left(\mathrm{m}^{2}\right)$, and $\eta$ is the efficiency that corresponds to the electric quantity produced as a percentage of the received solar power. We assume that this power is used for charging EVs. This generated power highly depends on irradiation and cloudiness index as they differ during seasons and day hours. For example, PV production is maximal in a sunny day during Summer at noon. The actual values of irradiation at different seasons and day hours are fixed, and thus, known in advance ${ }^{3}$ Based on real irradiation values, we estimate the amount of PV energy that can be produced by the considered PV farm during a sunny day at each month of the year (see Fig. 4 in appendix). We observe that up to $90 \mathrm{MWh} /$ day can be generated during summer months when irradiation is at its highest, while this production drops to approximately 30 $\mathrm{MWh}$ /day during winter. However, these values can be seen as the maximal daily production (i.e. calculated in fully-sunny days), and thus, will be reduced due to cloudiness effects. In addition, PV farms rely on their annual energy production more than power peaks and profiles throughout a day or a season. A high power consumption (charging) combined with a high PV energy production can thus create flow fluctuations to the grid unless they are well synchronized.

TABLE II: Cloudiness index - Paris Orly (2010-2020)

\begin{tabular}{|c|c|c|c|c|}
\hline & Winter & Spring & Summer & Autumn \\
\hline Fully-Sunny & $16.9 \%$ & $27.2 \%$ & $35.6 \%$ & $22.9 \%$ \\
Partially-Cloudy & $6.7 \%$ & $9.1 \%$ & $15.6 \%$ & $9.1 \%$ \\
Mostly-Cloudy & $11.4 \%$ & $17.6 \%$ & $21.5 \%$ & $11.6 \%$ \\
Fully-Cloudy & $65.1 \%$ & $46.1 \%$ & $27.3 \%$ & $56.2 \%$ \\
\hline
\end{tabular}

Unlike the irradiation, the cloudiness index $(\mu(t))$ can not be fixed in advance as it depends on weather conditions (i.e. $\mu(t)$ is stochastic). We thus need to model this uncertainty using a set of scenarios and calculate their probability distribution. We consider 4 different day profiles: (1) Fully-Sunny, where $\mu(t)$ is between $0 \% \& 25 \%$, (2) Partially-Cloudy, where $\mu(t)$ is between $25 \%$ \& $50 \%$, (3) Mostly-Cloudy, where $\mu(t)$ is between 50\% \& 75\%, and (4) Fully-Cloudy, where $\mu(t)$

\footnotetext{
${ }^{3}$ We use data provided by the Photo-voltaic Geographical Information System (PVGIS) to build the irradiation matrix for Paris-Saclay area
}

is between $75 \%$ \& $100 \%$. In addition, we divide the day into 8 time periods (i.e. (12AM,3AM), (3AM,6AM), .. , (9PM,12AM)). These day periods help in capturing different mobility patterns (e.g. period (6AM-9AM) is a peak period when people commute, etc.). We also consider the four seasons (e.g. the probability of having a fully-sunny day is higher in Summer than in winter, Table III). As a result, we build our set of scenarios, where every scenario is defined by a day-profile, a day-period, and a season. In order to calculate their probabilities, we use weather measurements of the last 10 years and we cluster them according to day profiles, periods, and seasons using K-Means approach ${ }^{4}$. The resulting probability distribution represents an input to our model.

\section{B. Deploying EV fast-chargers}

We test the proposed model using Paris-Saclay data in order to obtain the optimal deployment of EV charging stations in the area. Results indicate the number of chargers to be installed at each selected location $\left(\left(z_{s}^{a}, z_{s}^{b}\right)\right.$ in Fig. 3). It can be observed that the number of vehicle chargers is relatively higher than that for trucks at all selected locations. This can be explained by the higher number of vehicles to be recharged at different paths. Another important observation is that the proposed deployment can cover up to $33.1 \%$ of the overall EV charging demand. More precisely, some paths are fullycovered (e.g. "A126" with 100\%), some are partially-covered (e.g. "A10" and "N118" with $38 \%$ and $64 \%$ respectively), and some others are poorly-covered (e.g. "A6" and "N104" with only $3.8 \%$ and $8.3 \%$ respectively). Low coverage rates at some paths are due to the small number of charging locations and the limited electric capacity at these locations.

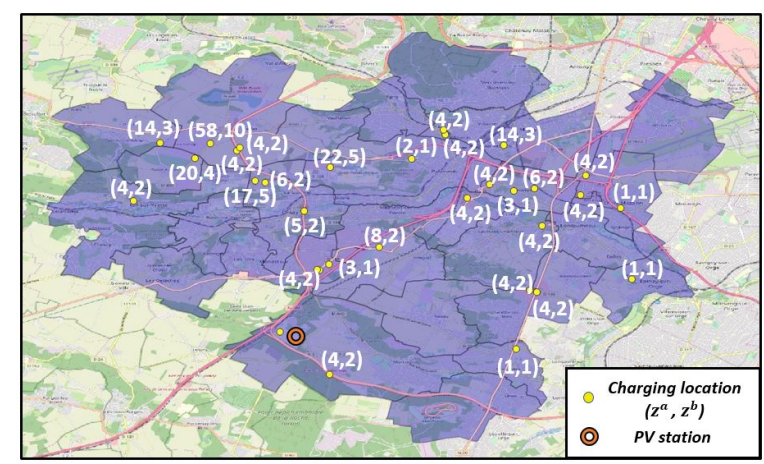

Fig. 3: Number of chargers per station

TABLE III: Demand coverage with PV production per season

\begin{tabular}{|c|c|c|c|c|c|}
\hline & No PV & PV-Wint. & PV-Spr. & PV-Sum. & PV-Aut. \\
\hline Overall & $33.1 \%$ & $37.3 \%$ & $40.9 \%$ & $44.4 \%$ & $39.1 \%$ \\
A10 & $3.8 \%$ & $19.9 \%$ & $33.5 \%$ & $47.1 \%$ & $27.1 \%$ \\
N104 & $8.3 \%$ & $29.9 \%$ & $53.3 \%$ & $74.2 \%$ & $39.8 \%$ \\
\hline
\end{tabular}

${ }^{4}$ Weather data for Paris-Orly airport, including cloudiness index, are provided by Météo France 
As introduced earlier, the considered PV farm is located at the intersection of "A10" and "N104" which are poorlycovered paths. Thus, integrating its produced energy has the potential of enhancing the coverage rates at these paths as more chargers can be installed. To analyze this potential benefit, we quantify the amount of energy that can be produced at different scenarios and we calculate the demand coverage rate at each case. We then compare the obtained rates to the case where no PV production is considered (Table III). By averaging 10 different runs of the model, we observe that coverage rates can increase from $3.8 \%$ and $8.3 \%$ to $47.1 \%$ and $74.2 \%$ respectively during Summer where PV production is maximal. This positive impact can also be witnessed on the overall coverage rate with approximately $7 \%$ to $11 \%$ increase in Spring and Summer scenarios. As a result, locally-produced PV energy can help in enhancing the quality of EV charging service especially along paths where the demand is high and electric grid is limited.

\section{Analyzing energy prices and production costs}

We study three different investment indices to evaluate the profitability of integrating PV energy. First, Levelized Cost of Energy ( $L C O E$ ) which represents the average net cost of electricity generation for the planned PV farm over its lifetime [6]. It can be calculated as the ratio between lifetime costs and energy production, as follows:

$$
L C O E=\frac{\sum_{t=1}^{n} \frac{I_{t}+M_{t}+F_{t}}{(1+r)^{t}}}{\sum_{t=1}^{n} \frac{E_{t}}{(1+r)^{t}}}
$$

Where $I_{t}, M_{t}$, and $F_{t}$ are the investment, maintenance, and fuel costs in year $t, E_{t}$ is the electricity generation in year $t$, and $r$ is the discount rate. Second, Return on Investment (ROI) which is used to evaluate the efficiency of an investment by measuring its amount of return [7]. It is calculated by dividing project net income to its total cost, as follows:

$$
R O I=\frac{\text { Project return }(\text { income }- \text { cost })}{\text { Project cost }} \times 100
$$

Third, Payback Period (PP) which represents the amount of time needed to recover the cost of an investment. It can be calculated by dividing overall project investment to its annual profit, as follows:

$$
P P=\frac{\text { Investment amount }}{\text { Annual profit }}
$$

The project of installing solar panels at Paris-Saclay area requires an investment of $20 \mathrm{M} €$. The investment costs $\left(I_{t}\right)$ appear only in the first year as they represent the cost of installing solar panels and EV fast-chargers (estimated at $870 \mathrm{~K} €$ based on the number of chargers proposed by the model). Maintenance costs $\left(M_{t}\right)$ of a PV farm appear every 3 years and are estimated to $10 \%$ of the annual profit 5 Fuel

\footnotetext{
${ }^{5}$ National Center of Photovoltaic Resources (CRPV)
}

costs $\left(F_{t}\right)$ are ignored in this case and the annual electricity generation $\left(E_{t}\right)$ is estimated at $24 \mathrm{GWh}$ (i.e. we assume that all generated PV energy is used for charging EVs). Discount rate $(r)$ is fixed at $5 \%$. In addition, project income is the difference between $\mathrm{PV}$ production cost (i.e. the calculated $L C O E$ ) and the price of selling this energy for charging EVs. In France, the price of charging an EV using a Tesla super-charger is $0.2 € / \mathrm{kWh}$. Thus, the cost of generating a kilowatt at the PV station must be less than $0.2 €$ in order to have a positive income.

TABLE IV: PV energy - Cost analysis

\begin{tabular}{|c|c|c|c|}
\hline & 10 years & 15 years & 20 years \\
\hline Levelized Cost of Energy (LCOE) & 0.11 & 0.08 & 0.07 \\
Return on Investment (ROI) & $148 \%$ & $224 \%$ & $260 \%$ \\
Payback Period (PP) & 9.66 & 7.39 & 6.65 \\
\hline
\end{tabular}

PV farms are often evaluated over 20 years lifetime [7]. However, we calculate the introduced indices on 10, 15, and 20 years lifetime (Table IV). Results show that net cost of the generated PV energy $(L C O E)$ is estimated at $0.11,0.08$, and $0.07 € / \mathrm{kWh}$ over different lifetime intervals. These values can be seen as the minimum cost required for selling electricity at break-even prices so that project expenses can be recovered during its lifetime. Relatively, the planned investment brings a positive return of $260 \%$ over a 20 years lifetime. This means that every $100 €$ of investment in the considered PV farm will be returned as $260 €$ at the end of its lifetime. These positive $R O I$ values justify the benefits of using the locallyproduced PV energy for supplying EV chargers. Regarding the refund time of investment $(P P)$, results indicate the considered investment will be able to pay itself back in approximately 7 years, which is less than half of the project lifetime.

\section{CONCLUSION}

In this paper, an optimization model for the deployment of EV fast-charging stations through a network of highways has been introduced. The model has been tested using a Cplex solver and a case study on Paris-Saclay area has been performed. After selecting optimal locations for charging stations, the results highlighted the benefits of integrating PV energy production on improving demand coverage rates. In addition, the economical benefits of this integration has been quantified ( $R O I=260 \%$ over 20 years lifetime). We believe that this study helps in a better understanding of the potential use of green energy sources for satisfying the increasing charging demand for electric vehicles.

\section{ACKNOWLEDGMENT}

This research work is carried out within the PGMO research project at GeePs laboratory, CentraleSupélec. The work is in collaboration with the Institute of Research and Technology SystemX and the Agglomeration Community of Paris-Saclay. 


\section{REFERENCES}

[1] A. Mourad, J. Puchinger, and C. Chu, A survey of models and algorithms for optimizing shared mobility, Transportation Research Part B: Methodological, vol. 123, pp. 323-346, 2019.

[2] B. Robertson, J. Bekker, and B. Buckham, Renewable integration for remote communities: Comparative allowable cost analyses for hydro, solar and wave energy, Applied Energy, vol. 264, pp. 114677, 2020.

[3] H. Shareef, MD. Mainul Islam, and A. Mohamed, A review of the stageof-the-art charging technologies, placement methodologies, and impacts of electric vehicles, Renewable and Sustainable Energy Reviews, vol. 64, pp. 403-420, 2016

[4] Y. He, K. M. Kockelman, and K. A. Perrine, A Optimal locations of U.S. fast charging stations for long-distance trip completion by battery electric vehicles, Journal of Cleaner Production, vol. 214, pp. 252-461, 2019.

[5] Z. Sun, W. Gao, B. Li, and L. Wang, Locating charging stations for electric vehicles, Transport Policy, pp. 1-7, 2018.

[6] F. A. Khan, N. Pal, and S. H. Saeed, Review of solar photovoltaic and wind hybrid energy systems for sizing strategies optimization techniques and cost analysis methodologies, Renewable and Sustainable Energy Reviews, vol. 92, pp. 937-947, 2018.

[7] O. Ozcan, and F. Ersoz, Project and cost-based evaluation of solar energy performance in three different geographical regions of Turkey: Investment analysis application, Engineering Science and Technology, an International Journal, vol. 22, pp. 1098-1106, 2019.

[8] C. Good, M. Shepero, J. Munkhammar, and T. Boström, Scenario-based modelling of the potential for solar energy charging of electric vehicles in two Scandinavian cities, Energy, vol. 168, pp. 111-125, 2019.

[9] D. Thomas, O. Deblecker, and C. S. Ioakimidis, Optimal operation of an energy management system for a grid-connected smart building considering photovoltaics' uncertainty and stochastic electric vehicles' driving schedule, Applied Energy, vol. 210, pp. 1188-1206, 2018.

[10] S. A. Funke, F. Sprei, T. Gnann, and P. Plotz, How much charging infrastructure do electric vehicles need? A review of the evidence and international comparison, Transportation Research Part D: Transport and Environment, vol. 77, pp. 224-242, 2019.

\section{APPENDIX}

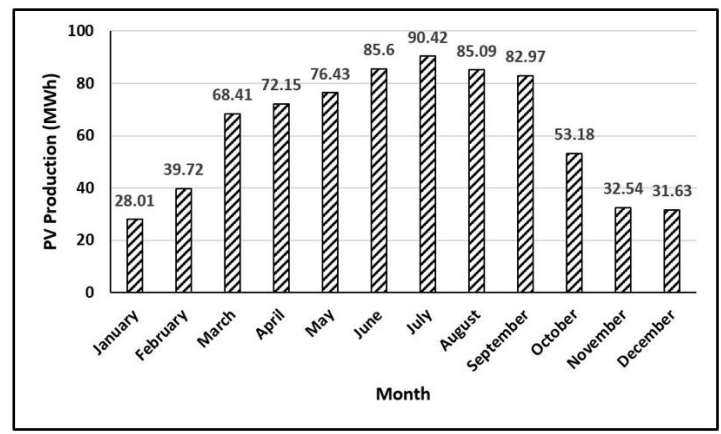

Fig. 4: Maximal daily PV production per month 